\title{
STUDY OF DATA MINING TECHNIQUE IN COLLIERY EQUIPMENTS FAULT DIAGNOSIS
}

\author{
Hongxia Pan, Jinying Huang, Zonglong Xu \\ College of mechanical engineering and automatizition, North University of China, China; \\ Email: panhx1015@yahoo.com.cn.
}

Abstract: In this paper, aiming at the fault phenomenon of many colliery electromechanic equipments can't be expressed with the structural data, and the traditional expert system that based on the rule reasoning is very difficult to extracting the rule, put forward a kind of method that regard the numerous diagnosis case examples in the past and the faults that possibly occur and the elimination project as the knowledge source. It set up a structure frame of the data mining system that was adopted in the fault diagnosis of the colliery equipments, which based on the association rule, and discuss the data mining based on the association rules of the single layer fault and the multilayer fault in the colliery equipments system.

Key words: Data Mining, Fault Diagnosis, Colliery Equipments, Association Rule.

\section{INTRODUCTION}

Along with the flying development of science and technology, the openair excavate coal equipments have gained a fast renewal. Adopting the efficient importing equipments largely, heightened the automation degree and the production efficiency of the excavate coal. At the same time, because of the structure complexity and function perfectness of the excavate coal electromechanic equipments, after they are used several years, the multifarious faults begin to occur continually. Therefore, to carry on the efficient and fast fault diagnosis for these importing electromechanic equipments, and keep its good usage performance, is an important assurance to heighten the production efficiency and reduce the cost. However, to carry

This project is supported by the National Natural Science Foundation of China under the grant No.50575214.

Please use the following format when citing this chapter:

Pan, Hongxia, Huang, Jinying, Xu, Zonglong, 2006, in International Federation for Information Processing (IFIP), Volume 207, Knowledge Enterprise: Intelligent Strategies In Product Design, Manufacturing, and Management, eds. K. Wang, Kovacs G., Wozny M., Fang M., (Boston: Springer), pp. 328-333. 
on the sufficient exposure of fault source and ascertaining the fault position for the numerous and such complicated equipments, only depending on the diagnosis and maintenance man who used the traditional methods to carry on the fault diagnosis and exclusion, which has more localization generally. To introduce the artificial intelligence into the fault diagnosis field of the excavate coal equipments, which provides a new method for the fault diagnosis of the excavate coal equipments.

In this paper, aiming at the characteristic of the excavate coal equipments, which the numerous parts of which the performance is complicated and each constitute a complicated system, researched to make use of the data mining technique to obtain the association rule between fault and reason. It makes the decision maker easily get the fault relating degree among each subsystem of the excavate coal equipments. It provides the important reference information for fleetly and thoroughly eliminating faults..

\section{DATA MINING TECHNIQUE IN EQUIPMENTS FAULT DIAGNOSIS}

The data mining technique is one of the important researched contents of the intelligence system theory and technique nowadays ${ }^{[1]}$. It refers multidomain - machine learning, pattern recognition, inductive reasoning, statistics, database, data viewable, high performance calculation, etc. The data mining technique aims at discovering the knowledge concealed in the numerous data, and will be applied to resolving the problem that is "data rich but knowledge poor". It has been applied to some trades-industry, business, finance, medicine, administration management, communicate network, etc. It has played a pole in some ways, such as the modeling of the fuzzy controller, the modeling and forecasting of fault diagnosis, etc.

In the fault diagnosis of equipments, it is the key step to build the diagnosis knowledge model. The knowledge model of the diagnosed equipments is expressed by 4 kinds of modes, such as the equipments running model, the diagnosis experience rule, the diagnosis decision model and the diagnosis case. According to the diagnosis knowledge sequence from shallow to deep and from general to special, they can be divided into four layers to be organized. The first layer knowledge describes the elementary diagnosis knowledge, providing the essential elucidation for the diagnosis. The second layer knowledge describes the general diagnosis knowledge, applying to the diagnosis that based on rules. The third layer knowledge is the diagnosis decision model that was formed according to the similar diagnosis case via training and structuring. The fourth layer knowledge is 
consisted of the case database that based on the binary tree, applying to the diagnosis that based on cases ${ }^{[2]}$.

\subsection{Build self-learning model of fault diagnosis knowledge}

The strategy of the modeling is: (1)Learn the diagnosis rule between the symptom and the diagnosis result from dataset. (2) Carry on testing and processing, every data sample that can be explained by mined diagnosis rule should be deleted from dataset. Regard the dataset as the training sample set, applying to build the diagnosis decision model; (3)After structuring the decision model via the training and leaning, carry through testing and processing for the dataset, every data sample that the error of diagnosis result is less than a certain threshold should be deleted from the dataset. (4) A small quantity of data that haven't been deleted in the dataset, after getting rid of the noise data via verifying mutually by users, are deposited in the case base. These cases express the diagnosis knowledge of special fault that is different with generic diagnosis knowledge model, and these cases carry on the organization of binary tree according to its exceptional characteristics, in order to carry on the fault diagnosis that based on cases.

\subsection{Constructing fault diagnosis model based artificial intelligence knowledge}

To constitute the fault diagnosis model of the fault diagnosis example set of diagnosis equipments via training, the form of model lies on the structure of model and its learning rule. They can be selected according to the instance of diagnosis equipments. It may be the Nerve Net model, or may be the Fuzzy Clustering model or other diagnosis models. After completing model training, can use the diagnosis models that have been built to compute the corresponding diagnosis result according the actual symptoms of diagnosed equipments.

\subsection{Fault diagnosis reasoning based on mixed knowledge model}

After building fault diagnosis knowledge models of the diagnosed equipments via self-training, can apply mixed knowledge model (function model, rule, decision model, case) to carry on fault diagnosis reasoning. Its strategy is: First, confirm the symptom according to the function model of diagnosed equipment, and carry on the diagnosis reasoning matching the corresponding fault diagnosis rule. If without the promise that fault diagnosis matched with the symptom, use the diagnosis decision model to carry on 
Diagnosis

fault diagnosis. If the precision of the diagnosis result of decision model is under the given threshold, then carry on the fault diagnosis that based on cases according to the symptom of diagnosed equipments matching with the diagnosis cases in case base.

\section{EQUIPMENT FAULT DIAGNOSING ANALYSIS BASED ASSOCIATION RULE}

\subsection{Building database of colliery equipments fault [3]}

Colliery equipments fault has layer, correlation and synthesis. Layer is that low-layer fault must induce high-layer fault, but high-layer fault can be induced by low-layer fault. Correlation is that after a certain structure cell has occurred faults, certainly will induce the correlative structure cell or correlative state with it to be changed, further will be likely to induce the correlative structure cell or correlative state to happen faults too. Synthesis is that any of primary faults has multifarious potential evocable faults. This shows, the colliery equipment is a system that many faults occur simultaneously. In this paper, the colliery electromechanic equipments are divided into four great systems - machinery and hydraulic subsystem, lubricating and cooling subsystem, electric and control subsystem, power drive subsystem. Such as the power drive subsystem, to build the fault database of power drive subsystem, as Table 1 showed.

Table 1. Fault database of power drive subsystem

\begin{tabular}{ccccc}
\hline Engine assembly & Fault 1 & Fault 2 & $\ldots$ & Fault k \\
\hline Turbine supercharger & Fault 1 & Fault 2 & $\ldots$ & Fault $\mathrm{m}$ \\
$\ldots$ & $\ldots$ & $\ldots$ & $\ldots$ & $\ldots$ \\
Gear-box assembly & Fault 1 & Fault 2 & $\ldots$ & Fault $\mathrm{n}$ \\
\hline
\end{tabular}

For every element of the subsystem database, can build its each database respectively as well as. Take example for engine assembly, can build its own fault database as Table 2 showed.

Table 2. Fault database of Engine assembly

\begin{tabular}{ccccc}
\hline Fault 1 & Cause 1 & Cause 2 & $\ldots$ & Cause $\mathrm{k}$ \\
\hline Fault 2 & Cause 1 & Cause 2 & $\ldots$ & Cause m \\
$\ldots$ & $\ldots$ & $\ldots$ & $\ldots$ & $\ldots$ \\
Fault k & Cause 1 & Cause 2 & $\ldots$ & Cause n \\
\hline
\end{tabular}




\subsection{Mining of association rule in single-layer fault}

The purpose of data mining rests with finding out the reliable and significative rules. Reliability predicates the reliable degree of rules. Support degree represents the important degree in all data. As a result, the association rule which reliability is small (that is the weak association rule) has a lower application value generally. After carrying through the mining of singlelayer association rule for the database of Table 1, can acquire such as association rules of the faults that occur in every constitute component of power drive subsystem. These association rules can afford the reference information that is worthily regarded for the elimination of relativity faults in single-layer equipments.

\subsection{Mining of association rule in multi-layer fault}

In the numerous faults that occur in every subsystem of colliery electromechanic equipments, many faults are often interactional and interrestricted. The rules of relational faults are concealed in the different abstract hierarchies of database, for example, the fault of clutch can cause all devices which were used in the subsystem of transmission back-end not to work normally. The fault logger of current equipments can't show the correlation of these faults, and numerous traditional fault diagnosis expert systems haven't the capability of finding out the correlation of multi-layer faults, the result can influence the comprehensive diagnosis of faults, therefore, it is considerably necessary and valuable for fault diagnosis to finding out the association rule of multi-layer faults.

Actually, all kinds of faults that occur in colliery equipments aren't often in the same layer, for example, engine system fault and clutch fault aren't in the same layer, but in fact clutch fault sometimes can cause the output of engine to change drastically, thereby induce engine system fault.

\section{STRUCTURE FRAME OF DATA MINING SYSTEM IN COLLIERY EQUIPMENTS FAULT DIAGNOSIS}

Basing on the analysis of the above application of association rule in colliery equipments fault diagnosis, in the colliery equipments fault diagnosis data mining system, its concept model of structural frame can be designed to be mainly composed of user query interface, query coordinator, database management module, knowledge base management module, model base management module, knowledge discover pretreatment module, knowledge evaluate module and conclusion interpret module etc. The structural frame of system is showed as Fig1. 
Diagnosis

\section{CONCLUSION}

Colliery equipment is a sort of system whose multi-fault is concurrent, and its fault has some characteristics, such as layer, relativity and syntheses etc., the traditional fault diagnosis expert system is not easy to extract the rule from the data structured difficultly. The data mining technique can mine the association rule on the base of the numerous fault data. It also can gain the valid association rule between the fault and the reason, as well as between faults. It provides some very important reference information for obviating the fault fast and thoroughly.

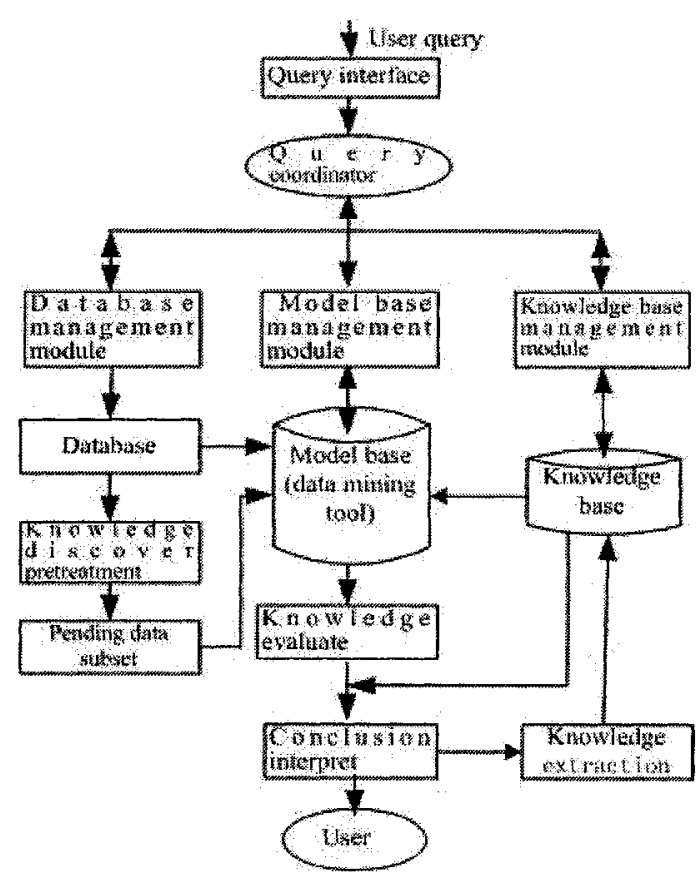

Figure1. Structure frame of data mining

\section{REFERENCES}

1. Fyyad U M, Piatetsky-shapiro G, Smyth P. Advances in knowledge discovery and data mining. California: AAAI/MIT, press, 1996.

2. Yang Jie, Huang Xin, LüYong. Data mining technique and its applications in modeling optimization and fault diagnosis [J]. Infrared and Laser Engineering, 2000,29(3):23-27.

3. Dong Doudou, Li Dengfeng, Chen Yuwen. Data Mining System Frame of Ship Fault Diagnoses based on Association Rules[J]. Ship Engineering, 2001,(4):61-64 